\title{
Measurement of antiproton production in p-He collisions and prospects for other inputs to cosmic rays physics from the fixed target program of the LHCb experiment
}

\author{
Giacomo Graziani* \\ on behalf of the LHCb collaboration \\ INFN Sezione di Firenze \\ E-mail: grazianiefi.infn.it
}

\begin{abstract}
The LHCb experiment has the unique possibility, among the LHC experiments, to be operated in fixed target mode, using its internal gas target SMOG. The energy scale achievable at the LHC and the excellent detector capabilities for vertexing, tracking and particle identification allow a wealth of measurements of great interest for cosmic ray physics. We present the first measurement of antiproton production in proton-helium collisions at $\sqrt{s_{\mathrm{NN}}}=110 \mathrm{GeV}$, which allows to improve the accuracy of the prediction for secondary antiproton production in cosmic rays. Prospects for other measurements achievable in the fixed target program are also discussed.
\end{abstract}

35th International Cosmic Ray Conference

10-20 July, 2017

Bexco, Busan, Korea

\footnotetext{
* Speaker.
} 


\section{Cosmic collisions in $\mathrm{LHCb}$}

The LHCb detector [1] is a single-arm forward spectrometer covering the pseudorapidity range $2<\eta<5$, designed for the study of particles containing $b$ or $c$ quarks, which are predominantly produced at high $\eta$ in $p p$ collisions at the LHC. The forward geometry and excellent vertexing, tracking and particle identification (PID) capabilities [2], which are key features for the reconstruction of heavy-flavour hadron decays, make it also an ideal tool to study interactions of the LHC beams with a fixed target. Such target is provided by the SMOG (System for Measuring Overlap with Gas) device [3, 4], through which tiny amounts of a noble gas ( $\mathrm{He}, \mathrm{Ne}, \mathrm{Ar}$ ) can be injected inside the primary LHC vacuum around the LHCb vertex detector (VELO). The design gas pressure in the VELO region is $2 \times 10^{-7}$ mbar, which is small enough not to significantly perturb the LHC operation. The device was originally conceived to determine the machine luminosity using a beam gas imaging technique[4]. Since 2015, LHCb has started to exploit SMOG to perform a set of physics runs, using special fills not devoted to $p p$ physics, with different beam and target configurations, allowing unique production studies which are relevant to cosmic ray physics. In particular, collisions of multi-TeV protons on helium at rest in $\mathrm{LHCb}$ reproduce the interactions of primary cosmic rays with the interstellar medium, which are expected to be the main source of cosmic ray antiprotons in the $10-100 \mathrm{GeV}$ energy range, where precise measurements have been achieved during the last years, notably by the AMS-02 [5] experiment. The interpretation of these results, which provide a sensitive indirect probe for Dark Matter, is currently limited by the accuracy of the predicted secondary antiproton flux. The largest uncertainty is due to the limited knowledge of the $\bar{p}$ production cross-section in the relevant processes. In particular, no data for $\bar{p}$ production in $p \mathrm{He}$ collisions existed so far.

\section{Measurement of antiproton production in pHe collisions}

LHCb performed the first measurement of $\bar{p}$ production in $p \mathrm{He}$ collisions by operating SMOG with helium during special fills with limited number of proton bunches, accelerated to $6.5 \mathrm{TeV}$ $\left(\sqrt{s_{\mathrm{NN}}}=110 \mathrm{GeV}\right)$. Most of the data were collected using a single LHC fill lasting about five hours in May 2016. Events were triggered with a minimum bias requirement, fully efficient on collisions producing an antiproton within the detector acceptance. The measurement is performed from collisions occurring in a $80 \mathrm{~cm}$ long fiducial region, along the beam direction $z$, where the best reconstruction efficiency is achieved. Antiprotons are counted in two-dimensional bins in momentum $(p)$ and transverse momentum $\left(p_{\mathrm{T}}\right)$, in the range $12<p<110 \mathrm{GeV} / c, 0.4<p_{\mathrm{T}}<4$ $\mathrm{GeV} / c$. The kinematic limits are dictated by the acceptance of the two ring-imaging Cherenkov detectors (RICH) providing particle identification. The first one covers the range $2<\eta<4.4$ and allows $\bar{p} / K^{-}$separation in the momentum range $10-60 \mathrm{GeV} / c$, while the second has acceptance $3<$ $\eta<5$ and actively identifies antiprotons between 30 and about $110 \mathrm{GeV} / c$. The analysis described in this document covers only the prompt $\bar{p}$ production, namely the antiprotons produced directly in the $p \mathrm{He}$ collision, or from resonances decaying via the strong interaction. The component due to hyperon decays, treated here as a background component and subtracted from the result, will be the subject of a dedicated study. 

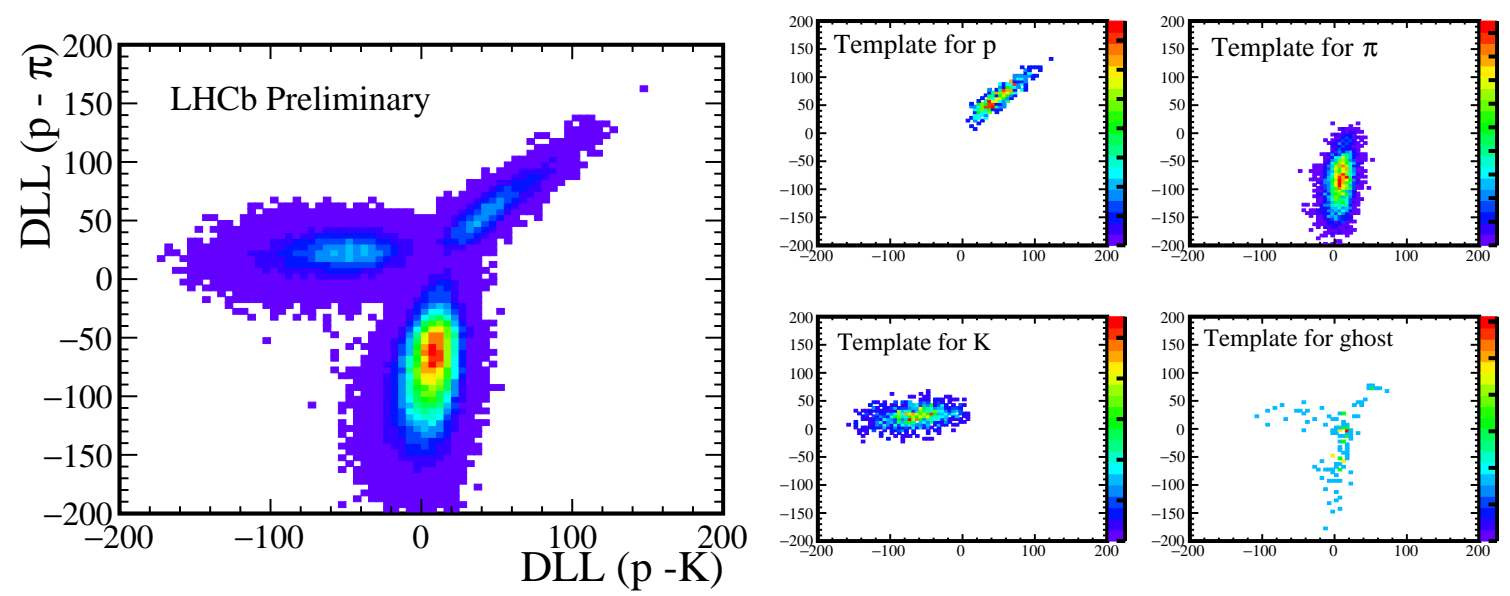

Figure 1: Example of two-dimensional DLL distributions for a particular bin $(21.4<p<24.4 \mathrm{GeV} / c, 1.2<$ $\left.p_{\mathrm{T}}<1.5 \mathrm{GeV} / c\right)$, illustrating the performance of the RICH detectors in separating $\pi^{-}, K^{-}$and $\bar{p}$ particles. The distribution in data is shown on the left, while the templates for the four categories, obtained from calibration samples (from simulation for the ghost component), are shown on the right.

\subsection{Reconstruction and particle identification}

Candidates are selected by requiring a negative track in the kinematic range of interest after applying quality requirements on the reconstruction of the track and of the collision primary vertex (PV). The PV reconstruction efficiency, estimated from simulation, varies with $z$ from $76 \%$ in the most upstream region to $95 \%$ around the nominal collision point, with a mild dependence on the $p_{\mathrm{T}}$ of the $\bar{p}$ candidate. The related systematic uncertainty is evaluated by weighting the simulation to account for discrepancies in the PV topology description with respect to data. The reconstruction efficiency for prompt antiprotons, $\varepsilon_{\text {rec }}$, including acceptance effects and the tracking detector efficiency, is determined from simulation in three-dimensional bins of $p, p_{\mathrm{T}}$ and $z$. The bins are chosen to be small enough to minimize the dependence on the assumed spectra in simulation, but are at least five times larger than the resolution in each variable. The size of the kinematic bins is chosen according to the expected spectrum of reconstructed antiprotons, while 12 bins of equal size are used for $z$. The tracking efficiency predicted by the simulation, averaged over $z$, ranges from 40 to $80 \%$ depending on the track kinematics. A correction determined from calibration samples in $p p$ data, of order $1 \%$, is applied to account for imperfections in the simulation of the tracking detector response.

Antiprotons within the selected sample of negative tracks are identified through the response of the RICH detectors, from which two variables are built, $\operatorname{DLL}(\mathrm{p}-\pi)$ and $\operatorname{DLL}(\mathrm{p}-\mathrm{K})$, representing the difference of the log likelihood between the proton and pion and the proton and kaon hypotheses. The fraction of antiprotons among the negative tracks is determined from the two-dimensional distribution of the DLL variables. Three sets of templates for the different particles species are considered: predicted by the simulation, obtained from calibration samples in the $p \mathrm{He}$ data set, and from calibration samples in the LHCb $p p$ data collected in 2016. The $p$ He calibration samples consist of selected $K_{\mathrm{S}}^{0} \rightarrow \pi^{+} \pi^{-}$decays for pions, $\Lambda \rightarrow p \pi^{-}\left(\bar{\Lambda} \rightarrow \bar{p} \pi^{+}\right)$for (anti)protons and $\phi \rightarrow K^{+} K^{-}$for kaons. The most appropriate calibration templates are chosen for each kinematic 
region, while the related systematic uncertainty is estimated from their comparison. The fraction of antiprotons in each kinematic bin of the selected sample is determined with a two-dimensional extended binned maximum likelihood fit, where the (DLL $(p-\pi), \operatorname{DLL}(p-K))$ distribution in data is fitted as the sum of four components: $\pi^{-}, K^{-}, \bar{p}$ and ghost tracks. The latter component, whose template is obtained from simulation, is needed to account for candidate tracks which can not be unambiguously matched to a particle. This occurs in simulation in about $2 \%$ of cases. The DLL distributions for data and calibration samples, illustrating the RICH performance, are shown in Fig. 1 for an arbitrary kinematic bin.

\subsection{Backgrounds}

The selected antiprotons that are not prompt are treated as a background and are subtracted from the selected sample. Such background is suppressed by requiring that the impact parameter (IP), which is reconstructed through the VELO with a resolution of $\left(15+29 / p_{\mathrm{T}}(\mathrm{GeV} / c)\right) \mu \mathrm{m}$, is compatible with zero. The residual nonprompt background varies in simulation between $3 \%$ at the lowest $p_{\mathrm{T}}$ values and $1 \%$ at high $p_{\mathrm{T}}$. In $90 \%$ of cases, this background is due to hyperon decays, while in the remaining cases the antiprotons come from a secondary collision in the detector material and are mistakenly assigned to the primary vertex. The average background level is constrained from the tail of the IP distribution in data to be $(2.6 \pm 0.6) \%$, where the uncertainty is systematic and is estimated by varying the fraction of nonprompt $\bar{p}$ in simulation within the range where a good agreement with data is observed.

Another background to be considered is due to the possible contamination of the gas target. The rate of collisions on the LHC residual vacuum is evaluated by acquiring part of the data without the injected helium gas, while using the same vacuum-pumping configuration of the data taking with gas. The yield measured in these special data are scaled according to the corresponding number of protons on target, and the relative average contribution from residual vacuum is evaluated to be $(0.7 \pm 0.2) \%$, where the uncertainty is systematic. Collisions on the residual vacuum exhibit a lower average track multiplicity than those on the helium target, indicating that the residual gas is mostly hydrogen, as indicated by rest gas analysis performed in the absence of beam.

\subsection{Normalization}

The SMOG device does not presently allow a precise calibration of the injected gas pressure. Instead, the normalization for the $\bar{p}$ production measurement is provided by observing a process with a well-known cross-section. Atomic electrons scattered off by the proton beam can be observed within the LHCb acceptance. For a $6.5 \mathrm{TeV}$ proton beam, in the corresponding kinematic range the scattering is purely elastic. The cross-section in the polar angle range $3<\theta<27 \mathrm{mrad}$, outside of which the electrons can not be reconstructed in LHCb, is $180.6 \mu \mathrm{b}$. Though this is three orders of magnitude below the total nuclear inelastic cross-section, events are expected to have a distinct signature, with a single low-momentum and low- $p_{\mathrm{T}}$ electron track visible in the detector, with little or no other activity. Background events which could mimic this signature are expected from soft nuclear interactions where the candidate electron is either the product of a photon conversion, or a light hadron from a central exclusive production event. In both cases the background is charge symmetric. This allows to model the background from events with a single positively charged track (referred to as single positrons in the following). Multiplicity distributions in data 

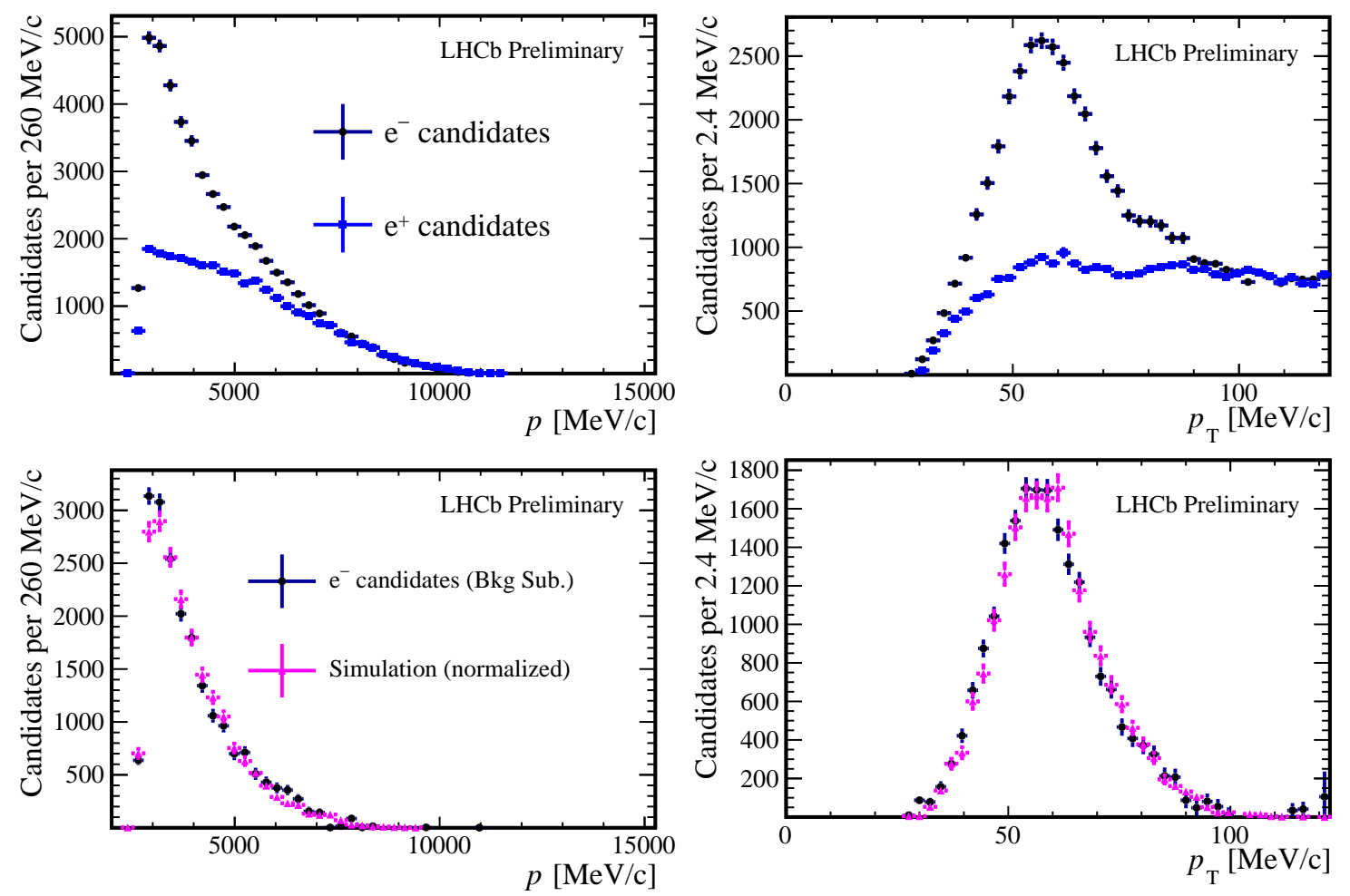

Figure 2: Distributions of (left) momentum and (right) $p_{\mathrm{T}}$ for (top plot) single electron and single positron candidates; (bottom plot) background subtracted electron candidates, compared with the distributions in simulation, which are normalized to data.

confirm that background-dominated regions close to the signal are charge symmetric. Single electron candidate events are selected through a loose kinematic selection on the track and applying veto requirements on any detector activity not compatible with the elastic scattering hypothesis. The selection yields 16569 single $e^{-}$candidates and $9548 e^{+}$candidates. The signal yield is obtained by the difference of the two components. The background subtracted kinematic distributions are shown on Fig. 2. An excellent agreement with the simulation is observed, confirming the validity of the charge-symmetry hypothesis for the background.

The luminosity is determined from the background-subtracted yield of scattered electrons $N_{e}$, the known cross-section $\sigma_{p e^{-}}$and the electron reconstruction efficiency $\varepsilon_{\mathrm{e}}$, as $\mathscr{L}=N_{e} /\left(Z_{H e} \times\right.$ $\sigma_{p e^{-}} \times \varepsilon_{\mathrm{e}}$ ), where $Z_{H e}=2$ is the helium atomic number. Possible effects of gas ionization were evaluated and are expected to be negligible. The reconstruction efficiency, evaluated from simulation, is limited by the soft momentum and transverse momentum spectrum. Electrons lose a sizable fraction of their energy through bremsstrahlung in the beam pipe and detector material, and large acceptance effects are caused by the spectrometer magnetic field. The relative systematic uncertainty on $\varepsilon_{\mathrm{e}}$ is estimated to be $6 \%$ from the stability of the result when varying the main selection criteria, and in particular from the ability of the simulation to describe the large modulation of the efficiency with the electron azimuthal angle. The result is $\mathscr{L}=0.443 \pm 0.011 \pm 0.027 \mathrm{nb}^{-1}$, where the first uncertainty is statistical, and the second is systematic, dominated by the uncertainty on $\varepsilon_{\mathrm{e}}$. 


\subsection{Uncertainties and results}

The precision of the measurement is limited by the systematic uncertainty. The largest uncertainty which is correlated among all kinematic bins is the aforementioned relative $6 \%$ on the normalization. The uncorrelated uncertainty is dominated for most bins by the error on the $\bar{p}$ fraction from the PID analysis. Large relative uncertainties, up to $26 \%$, affect the bins at the borders of the detector acceptance and, for the intermediate momentum region, in the transition region between the two RICH detectors, at $\eta \sim 4$.4. For the other regions, the accuracy is typically a few per cent. The relative total uncertainty amounts to $10 \%$ or less for most of the accessible $p_{\mathrm{T}}$ regions.

The antiprotons candidates are counted from a sample of 33.7 million selected $p \mathrm{He}$ collisions, from which a sample of 1.4 million antiprotons is determined by the PID analysis. The double differential $\bar{p}$ production cross-section $d^{2} \sigma / d p d p_{\mathrm{T}}$ is computed in each kinematic bin after correcting for the reconstruction efficiency and the background. The results are compared in Fig. 3 with the predictions of the EPOS LHC [6] model, which is used in the LHCb simulation. The double differential shape, notably the momentum spectrum, is found to be in good agreement with the simulation, while the absolute production rate is larger on average by about a factor 1.5. The data are also compared with three other models implemented in the CRMC [7] package v1.5.6: EPOS 1.99 [8], HIJING 1.38 [9] and QGSJET-II-04 [10].

The total inelastic cross-section is also determined from the measured total yield of recorded collisions within the fiducial region. The result is $\sigma_{\text {inel }}^{\mathrm{LHCb}}\left(p \mathrm{He}, \sqrt{s_{\mathrm{NN}}}=110 \mathrm{GeV}\right)=(140 \pm 10) \mathrm{mb}$ which is larger than the EPOS LHC prediction by a factor $1.19 \pm 0.08$, implying that the measured $\bar{p}$ multiplicity per inelastic collision is significantly larger in data. The multiplicity predicted by the pre-LHC version of EPOS is in better agreement with data. HIJING predicts a lower inelastic cross-section (100 mb), while it reproduces well the measured absolute $\bar{p}$ production cross-section values. QGSJET-II-04 exhibits a harder $p_{\mathrm{T}}$ spectrum than data, while the low energy extension QGSJET-IIm [11] is found to be in much better agreement with this measurement.

\section{Prospects}

The LHCb experiment has opened the way to the use of the LHC beams for fixed target physics. The first measurement of antimatter production in $p \mathrm{He}$ collisions is one of the first results of this novel program. Further details on this work can be found in the related LHCb conference contribution [12]. Developments of this study in the near future is foreseen, with the inclusion of the contribution due to hyperon decays, accounting for $20-30 \%$ of the $\bar{p}$ production. The measurement will be also performed on additional data collected at $\sqrt{s_{\mathrm{NN}}}=86.6 \mathrm{GeV}$ during November 2016, allowing to constrain scaling violation in the computation of the secondary antiproton flux in cosmic rays.

The production studies can be extended to other light particles, pion and kaons, allowing to predict also the positron production cross-section. Ratios of particle species, which are not affected by the uncertainty on the normalization, will provide precise tests of soft QCD models.

Production of exclusive charm states in the fixed target configuration gives access to the charm PDFs at large Bjorken- $x$ in the target nucleus, with unique sensitivity to antishadowing effects and to a possible contribution from intrinsic charm (IC). This can have a strong impact on the predicted 


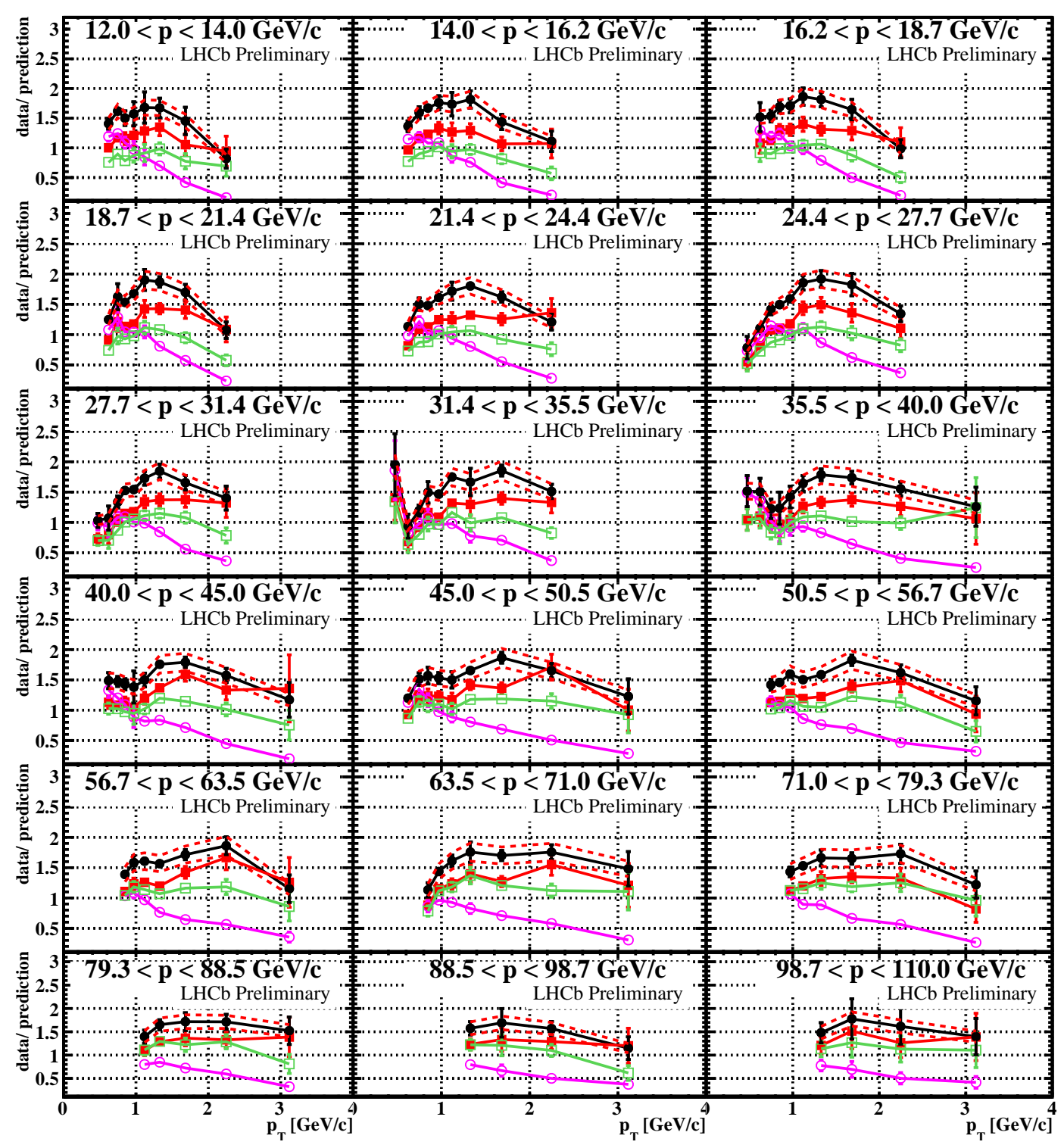

Figure 3: Result for the $\bar{p}$ cross-section measurement, compared to absolute predictions from different models. The plots show the ratio of data over simulation as a function of $p_{\mathrm{T}}$ in the 18 momentum bins, for (black round closed symbols) EPOS LHC, (red squared closed symbols) EPOS 1.99, (green squared open symbols) HIJING 1.38, and (violet round open symbols) QGSJETII-04. The error bars represent the uncorrelated uncertainty for each measurement. The additional correlated uncertainty, shown only for EPOS LHC but also relevant to the other cases, is indicated by the red dashed lines.

high-energy neutrino flux produced by cosmic atmospheric showers [13]. The first results for charm production, obtained from a data set of proton-argon collisions at $\sqrt{s_{\mathrm{NN}}}=110 \mathrm{GeV}$ corresponding to a few $\mathrm{nb}^{-1}$, have been recently released [14]. Though the results are still limited by the data size, the observed differential $D^{0}$ and $J / \psi$ yields are already expected to provide constraints on the IC contribution.

LHCb is also studying the possibility to identify light nuclei, to search for production of anti- 
deuterons and anti-helium in the collected data samples. The RICH detectors allow the identification of deuterons with momentum larger than about $20 \mathrm{GeV} / c$. For lower momentum, the use of $d E / d x$ and time-of-flight information from the tracking detectors is being investigated.

The collaboration is working on possible upgrades of the fixed target device. A precisely calibrated pressure gauge is being developed, aiming at reducing the uncertainty on the luminosity in the fixed target configuration.

\section{Acknowledgments}

We are indebted to our colleagues from the cosmic ray community, O. Adriani, L. Bonechi, F. Donato and A. Tricomi, who suggested this measurement, and would like to thank F. Donato and T. Pierog for their advice.

\section{References}

[1] LHCb collaboration, A. A. Alves Jr. et al., The LHCb detector at the LHC, JINST 3 (2008) S08005

[2] LHCb collaboration, R. Aaij et al., LHCb detector performance, Int. J. Mod. Phys. A30 (2015) 1530022

[3] C. Barschel, PhD thesis, RWTH Aachen U., 2014, Presented 05 Mar 2014

[4] LHCb collaboration, R. Aaij et al., Precision luminosity measurements at LHCb, JINST 9 (2014) P12005

[5] AMS collaboration, M. Aguilar et al., Antiproton Flux, Antiproton-to-Proton Flux Ratio, and Properties of Elementary Particle Fluxes in Primary Cosmic Rays Measured with the Alpha Magnetic Spectrometer on the International Space Station, Phys. Rev. Lett. 117 (2016) 091103

[6] T. Pierog et al., EPOS LHC: Test of collective hadronization with data measured at the CERN Large Hadron Collider, Phys. Rev. C92 (2015) 034906

[7] T. Pierog, C. Baus, and R. Ulrich, CRMC (Cosmic Ray Monte Carlo package), web.ikp.kit.edu/rulrich/crmc.html

[8] T. Pierog and K. Werner, EPOS Model and Ultra High Energy Cosmic Rays, Nucl. Phys. Proc. Suppl. 196 (2009) 102

[9] M. Gyulassy and X.-N. Wang, HIJING 1.0: A Monte Carlo program for parton and particle production in high-energy hadronic and nuclear collisions, Comput. Phys. Commun. 83 (1994) 307

[10] S. Ostapchenko, Monte Carlo treatment of hadronic interactions in enhanced Pomeron scheme: I. QGSJET-II model, Phys. Rev. D83 (2011) 014018

[11] M. Kachelriess, I. V. Moskalenko and S. S. Ostapchenko, New calculation of antiproton production by cosmic ray protons and nuclei, Astrophys. J. 803 (2015) no.2, 54

[12] LHCb collaboration, Measurement of antiproton production in $p$ He collisions at $\sqrt{s_{\mathrm{NN}}}=110 \mathrm{GeV}$, CERN-LHCb-CONF-2017-002

[13] R. Laha and S. J. Brodsky, IC at IC: IceCube can constrain the intrinsic charm of the proton,arXiv:1607.08240 [hep-ph]

[14] LHCb collaboration, Measurement of $J / \psi$ and $D^{0}$ production in $p$ Ar collisions at $\sqrt{s_{N N}}=110 \mathrm{GeV}$, CERN-LHCb-CONF-2017-001 